\title{
La bioética del sistema CRISPR-Cas9 como terapia génica en enfermedades de importancia mundial
}

\author{
The bioethics of the CRISPR-Cas9 system as genetic therapy for globally-important illnesses
}

\author{
Marifer Hernández-Hernández, ${ }^{a}$,Alma Barajas-Espinosa ${ }^{b}$
}

\begin{abstract}
:
Genetics, the field of study of hereditable material that dictates individuals' phenotype, has greatly evolved since 1953, the year in which this material's basic structure was published. The advances have been of such magnitude that today we can easily localize human genes, access their entire sequence and even pinpoint genes involved in human disease development. Cutting edge techniques can be applied to modify and improve our food supply, be they of vegetable or animal origin. Genetic engineering techniques have been applied with the objective of solving global-scale social problematics, including the high index of morbidity and mortality without having factored in social factors, public health problematics, and the ethical implications of the techniques. Notwithstanding the recognized importance of ethics as an integral necessity in the medical practice, strict application of bioethics, however, has also complicated scientific innovation, due to the series of norms and regulations. In this essay we will revise the bioethics behind the CRISPR-Cas9 system as a genetic therapy tool for globally-important illnesses.
\end{abstract}

\section{Keywords:}

CRISPRCas, genetics, engineering, ethics

\section{Resumen:}

Genética, el estudio del material hereditario que dicta el fenotipo de individuo, ha evolucionado mucho desde el año 1953 cuando se publicó la estructura básica de este material. El avance ha sido de tales magnitudes que hoy día podemos fácilmente localizar los genes del humano, obtener su secuencia completa, e incluso encontrar genes relacionados al desarrollo de enfermedades humanas. Inclusive hemos sido capaces de utilizar técnicas de punta para modificar y mejorar nuestros alimentos, de origen animal o vegetal. La aplicación de técnicas de ingeniería genética se ha realizado bajo la presunción de resolver problemas sociales de grandes magnitudes como lo son los índices de morbilidad y mortalidad dentro de la población, sin necesariamente haber tomado en cuenta factores sociales, problemas de salud pública o las implicaciones éticas asociadas a su uso. No obstante, reconociendo la importancia de la moral dentro de la práctica médica, hay que también reconocer que en ocasiones la bioética ha complicado la innovación científica, debido a la complejidad de las normativas. En este ensayo se revisará las implicaciones bioéticas del uso del sistema CRISPR-Cas9 como terapia génica en enfermedades de importancia mundial.

\section{Palabras Clave:}

\section{Ensayo}

En el año 1953, Watson y Crick publicaron un comunicado científico que revolucionaria al mundo, la estructura del ADN, esa doble hélice que contiene toda la programación genética de cada individuo ${ }^{1}$ en un formato altamente sencillo, un código conformado por tan solo cuatro bases. La combinación de estas cuatro bases, en conjunto con un sistema celular complejo, es lo que permite que se produzcan el gran conglomerado de aproximadamente 6.13 millones de diferentes proteínas que constituyen la maquinaria del cuerpo humano. ${ }^{2} \mathrm{EI}$ proyecto del genoma humano comenzó en el año 1990 y concluyó en 2003, logró la secuenciación de más de 3.2 billones de bases. ${ }^{3}$ Este proyecto internacional ha sido fundamental para el desarrollo de la terapia génica; en la cual se interfiere con la expresión de genes, intentando una manipulación correctiva de la enfermedad a nivel molecular. Por lo tanto, la genética moderna ha tenido un gran avance en los últimos 17 años, con el desarrollo de 
técnicas de biología molecular para estudiar el genoma y sus funciones. La genética clínica, realizada con fines terapéuticos, es ejecutada sobre un gen afectado que no cumple su función adecuadamente, dando como consecuencia una enfermedad. Cabe aclarar que esta intervención es implementada con la finalidad de erradicar el problema que afecta a un individuo y no a su descendencia cuando se realiza en las células somáticas. ${ }^{4}$ Este proceso puede ser empleado en células, directamente dentro del organismo (in vitro e in vivo, respectivamente) y es introducida por medio de vectores, que pueden ser de tipo viral o plasmídico. ${ }^{5}$ El objetivo principal de la terapia genética es introducir a un sistema biológico un gen, capaz de ser trascrito y traducido, cuya permanencia sea prolongada, para que los efectos de dicha terapia sean efectivos en los pacientes afectados. Está terapéutica es realizada bajo la premisa "Primum non nocere", que significa "Lo primero es no hacer daño," en base a los criterios de investigación y normativas correspondientes. ${ }^{6}$ El sistema CRISPR/Cas 9 consiste de Repeticiones Palindrómicas Cortas Agrupadas $y$ Regularmente Inter espaciadas (por las siglas en inglés, CRISPR: Clustered Regularly Interspaced Short Palindromic Repeats) y de un sistema enzimático asociado (Cas9: CRISPR-associated system). Dicho sistema se encuentra funcionando de manera natural en aproximadamente el $50 \%$ de bacterias y funge un rol de protección en contra de la invasión viral. Las bacterias infectadas por un virus incorporan segmentos de secuencias del genoma viral en segmentos CRISPR de los genomas bacteriales. Esta incorporación permite a la bacteria reconocer subsecuentes infecciones virales y activar las nucleasas adecuadas para su destrucción, las "Cas." ${ }^{7}$ Las nucleasas son dirigidas de manera específica debido a la producción de un ARN complementario a la secuencia blanco, una característica que se ha utilizado para también dirigir de manera específica la escisión de secuencias de ADN mutadas en el genoma de células eucariotas, y estas son remplazadas por fragmentos de ADN funcionales. ${ }^{8}$ Dicho sistema de recortar y remplazar fragmentos de ADN se ha empleado en células humanas autosómicas ${ }^{9}$, células madre ${ }^{10}$, e incluso en células germinales ${ }^{11}$, afectando de tal manera la función de tejidos específicos. ${ }^{12}$ Pero, como Stan Lee escribió en el comic del Hombre Araña, "Un gran poder conlleva una gran responsabilidad." ${ }^{13}$ Interesantemente el Hombre Araña es un personaje basado en un individuo cuyo genoma es accidentalmente modificado. En el presente ensayo se mostrará información para concientizar sobre la necesidad medica de la terapia génica y la investigación del sistema CRISPR-Cas, los dilemas bioéticos que presenta el uso de este sistema $y$, finalmente, se postularan maneras de solventar el problema de la bioética del sistema CRISPR-Cas9 como terapia génica en enfermedades de importancia mundial. Según la OMS, a nivel mundial las cardiopatías y la Enfermedad Pulmonar Obstru ctiva Crónica (EPOC) son las principales causas de muerte, mientras a nivel nacional las principales cau sas lo son el cáncer, el infarto agudo al miocardio y la diabetes. ${ }^{14}$ Las organizaciones gubernamentales SSA, INEGI y CONAPO han llegado a la conclusión de que la situación epidemiológica en México se encuentra en niveles críticos debido a las condiciones en las que se desarrolla la población en general. ${ }^{15}$ Esta situación es dada principalmente por enfermedades con etiología multifactorial, que incluye factores genéticos y ambientales. ${ }^{16}$ Las causas genéticas, por ejemplo, asociadas con el desarrollo de EPOC son heterogéneas ${ }^{17}$ y en ciertos casos, la base genética solo explica un porcentaje bajo de la incidencia de la enfermedad, como es en el caso de la diabetes tipo 2. ${ }^{18}$ Sin embargo, tanto la genética y la exposición ambiental, contribuyen a generar una mayor tendencia a que el alelo afectado por disposición genética se exprese. Consecuentemente, los factores ambientales son un detonador importante para activar la expresión anómala de los genes relacionados a enfermedad. Entre los factores que aumentan la vulnerabilidad individual a desarrollar las enfermedades mencionadas se encuentra la situación económica, los altos niveles de contaminación y las condiciones en las que se vive y se trabaja. ${ }^{19}$ Debemos de tomar en cuenta que no solo el genoma humano cambia con el tiempo, si no que los mismos seres humanos creamos de forma indirecta esos cambios, que son transmisibles a las futuras generaciones. ${ }^{20}$ El primer ensayo clínico del sistema CRISPR-Cas9 en humanos, dirigido a corregir un receptor de linfocitos $\mathrm{T}$ en pacientes con cáncer, comenzó hace ya dos años y se está esperando los resultados

("https://clinicaltrials.gov/ct2/results?pg=1\&load=cart\&id= NCT03399448" n.d.). Actualmente, su aplicación en enfermedades respiratorias ${ }^{22}$, cardiopatías ${ }^{23}$ y diabetes ${ }^{24}$ se encuentra bajo escrutinio de la población científica. Un área que debe ser investigada a mayor profundidad son los efectos colaterales que dicha técnica pudiese tener en los mismos sitios blancos ${ }^{25} 0$ en sitios completamente inesperados. ${ }^{26}$ También es importante aumentar el conocimiento sobre el sistema CRISPRCas, en particular el mejor caracterizado para la edición genómica que es el tipo II, osea el CRISPRCas9. ${ }^{27} \mathrm{La}$ factibilidad de diversos blancos genéticos es discutida y estudiada en modelos celulares e inclusive en modelos animales ya que se han generado resultados muy alentadores en la aplicación de terapias génicas con diversas enfermedades metabólicas. ${ }^{28}$ A pesar de que esta alternativa promete resultados alentadores, la comunidad científica siempre se ha encontrado envuelta en dilemas éticos por los resultados no tan afines a sus propuestas de investigación experimental y que llegan a comprometer a la vida en general. Este tipo de acontecimientos dieron origen a la bioética, en $1970{ }^{29}$; disciplina encargada de dictar principios que aseguren el respeto por la vida y por tanto ligada a los derechos humanos. Esta ciencia, es la encargada de estipular las bases en las que se debe apoyar el investigador para regirse y a su vez sustentar sus acciones.

La terapia génica fue teorizada desde 1960 por Stanfield en un ensayo experimental referente a enfermedades congénitas del metabolismo, y aun cuando la terapia génica no existía en la práctica, rápidamente comenzaron a surgir problemáticas éticas ante su posibilidad. Se considera al CRISPR-Cas9 como una de las mejores opciones a la terapia génica para la edición en el panorama clínico, ya que resulta menos tóxico que sus predecesores: los dedos de zinc y el sistema TALEM, 
además de que resulta más económico que los procedimientos anteriores, otorgando una mejor calidad de vida al paciente. ${ }^{30}$ Existen varias preguntas éticas sobre el tema, como por ejemplo, ¿cómo decidimos cuando un gen es malo y debe ser reemplazado ${ }^{31} \mathrm{La}$ respuesta más directa es que consideramos a un gen como malo cuando causa enfermedad, o cuando se altera la función de un organismo fuera de lo normal. Sin embargo, mucho antes de conocer la estructura de los genes, se reconocía que la evolución de los organismos se realizaba gracias a un proceso de mutaciones ${ }^{32}$ sucesivas. Claro, debemos reconocer que, por lo menos en el corto plazo, no todas las mutaciones genéticas conllevan una adaptación al medio ambiente. La terapia génica fue aprobada en 1990 para su implementación en seres humanos, confirmado por parte de la Whiley Database on Gene Therapy Clinical Trials Wordlwide, del National Institute of Health $(\mathrm{NIH})$ de los Estados Unidos de America. Pero, no fue hasta el año 2000 en que se obtuvieron resultados positivos en su implementación como tratamiento del Adenosine deaminase severe combined immunodeficiency (ADA-SCID), tras diez ardorosos años de mejoramiento en la técnica. Otro acontecimiento significativo sucedió en el 2006, cuando la terapia génica fue empleada en el tratamiento de neoplasias obteniendo resultados importantes y significativos en estos pacientes. El "Proyecto de mil genomas" comenzó una vez consolidado el proyecto genoma humano en el 2003, y consistía en conocer las variantes entre individuos (polimorfismos) para conocer la expresión "normal" de genes entre poblaciones, tomando en cuenta su etnia, su localización geográfica y otros factores. Este fue la pauta que le dio un gran impulso para el apoyo y aprobación gubernamental para seguir avanzando en sus pruebas experimentales y ensayos clínicos, ya que demostró bases sólidas y otorgo bases comparativas. ${ }^{33}$ Todos estos aconteceres fueron generando polémica al ser una ciencia en desarrollo, su descubrimiento fue una esperanza de vida para los pacientes, pero no ha obtenido una aceptación total de la población en general pese a sus resultados. Hoy día, en el año 2020, cuando la terapia génica es un hecho factible, aplicado a pacientes con diversas aflicciones, surgen otro tipo de cuestiones éticas. Entre ellas, el costo elevado de dicha terapia y la limitada accesibilidad de la terapia a un grupo socioeconómico muy selecto. ${ }^{34} \mathrm{El}$ tremendo potencial de emplear la tecnología CRISPRCas para modificar células, tejidos, e incluso organismos enteros, conlleva al surgimiento de aún más numerosas cuestiones éticas, ya que el CRISPRCas provee un mecanismo de alterar la genética no solo en el individuo tratado, sino también en su descendencia. Por lo tanto, en cuestión de la terapia génica con CRISPRCas, su rgen dudas sobre: el grado en que esta debe ser permitida, su accesibilidad al público general, el tipo celular que se debe permitir ser utilizado, y la creación de leyes internacionales sobre su aplicación ${ }^{35}$. La terapia génica es una herramienta muy importante en la cura de enfermedades como el cáncer, ceguera, enfermedades respiratorias, enfermedades metabólicas, entre otras. La experimentación con personas o con tejido proveniente de humanos, se rige en nuestro país por la Ley General de Salud y el Reglamento de la Ley en Materia de
Investigación ${ }^{36}$ y sus reglamentos anexos, incluida la norma NOM-012-SSA3-2012 ${ }^{37}$ y la declaración de Helsinki ${ }^{38}$, en las cuales estipulan una serie de estatutos en los que se rige el comité de bioética con respecto a estudios preclínicos e investigaciones clínicas en seres humanos. Estos criterios enmarcan los principales estatutos a seguiry menciona las restricciones que deben de ser contemplado en la práctica. No obstante, sí bien es cierto que algunas instituciones creen irresponsable la experimentación en líneas germinales ${ }^{12}$, existen otras que creen pertinente la exploración y apertu ra de debates sobre si es correcto o no y la situaciones en que deben ser implementadas. ${ }^{39}$

En conclusión, la terapia génica es una rama de la medicina relativamente novedosa, que ofrece tratamien to dirigido y personalizado. El sistema de CRISPRCas, posee cualidades únicas que lo convierten en una herramienta valiosa en la terapia génica del humano. Los dilemas éticos planteados en contra de su implementación son claros y válidos, particularmente la cuestión de los efectos a largo plazo sobre la supervivencia de nuestra especie. El modificar a un organismo, para mejorar su fisiología, claramente es beneficioso para ese individuo y los que lo rodean. Sin embargo, los efectos que esta modificación pudiese tener en eventos de largo plazo, en términos evolutivos, se desconocen. Aun así, la modificación genética de nuestros alimentos se ha aprobado. ¿Por qué no aprobar la modificación genética en el humano? Mas aun, la terapia génica está realizándose en personas, pero solo en aquellos que pueden pagar una cuota inalcanzable (cerca de 2 millones de dólares) ${ }^{40}$ para la población humana en general. Entonces, ¿las cuestiones éticas de la terapia génica se omiten ante el poder socioeconómico? Queda claro que el poder de alterar la esencia biológica de un individuo es posible, pero solo es alcanzable por un grupo elite. Quizá la pregunta que se debe debatir es sobre la ética de la desigualdad de acceso al mejor tratamiento medicamente disponible, para así verdaderamente utilizar esta herramienta poderosa hacia la solu ción global de problemas de salud. Porque, como dijo James Watson, "antes pensábamos que nuestro futuro estaba en las estrellas, ahora sabemos que está en nuestros genes."

\section{Referencias}

1. Watson JD, Crick FHC. Molecular structure of nucleic acids: A structure for deoxyribose nucleic acid. Nature. 1953;171:737-738. doi: $10.1038 / 248765 \mathrm{a} 0$

2. Ponomarenko EA, Poverennaya EV., Ilgisonis E V., et al. The Size of the Human Proteome: The Width and Depth. Int J Anal Chem. 2016;2016. doi: $10.1155 / 2016 / 7436849$

3. The Human Genome Project | NHGRI. https://www.genome.gov/human-genome-project. Accessed March 24, 2020 .

4. Palacios-Yabar M. CRISPR / Cas9 : Edición Genética vs . Bioética. 2019;2(1):5-18. 
5. Athanasopoulos T, Munye MM, Yáñez-Muñoz RJ. Nonintegrating Gene Therapy Vectors. Hematol Oncol Clin North Am. 2017;31(5):753770. doi:10.1016/j.hoc.2017.06.007

6. Valenzuela CY. Ética científica de la terapia génica de individuos. Urgencia de la cirugía génica del ADN. Rev Med Chil. 2003;131(10):1208-1214. doi:10.4067/s0034-98872003001000018

7. Hille F, Richter H, Wong SP, Bratovič M, Ressel S, CharpentierE. The Biology of CRISPR-Cas: Backward and Forward. Cell. 2018;172(6):1239-1259. doi:10.1016/j.cell.2017.11.032

8. Giono LE. CRISPR/CAS9 Y LA TERAPIA GÉNICA. 2017;77:405409. www.medicinabuenosaires.com. Accessed March 24, 2020

9. Foltz LP, Howden SE, Thom son JA, Clegg DO. Functional a ssessment of patient-derived retinal pigment epithelial cells edited by CRISPR/Cas9. Int J Mol Sci. 2018;19(12). doi:10.3390/ijms19124127

10. Yumlu S, Bashir S, Stumm J, Kühn R. Efficient Gene Editing of Human Induced Pluripotent Stem Cells Using CRISPR/Cas9. In Methods in Molecular Biology. Vol 1961. Humana Press Inc. 2019:137-151. doi:10.1007/978-1-4939-9170-9_10

11. Callaway E. Errors in CRISPR-baby study. Nature. 2019;574:307.

12. Trapani I, Auricchio A. Seeing the Light after 25 Years of Retinal Gene Therapy. Trends Mol Med. 2018;24(8):669-681. doi:10.1016/j.molmed.2018.06.006

13. Roosevelt FD. Franklin D. Roosevelt's last message to the American people. Library of Congress. Presented at the: 1945

14. OMS | Mortalidad. https://www.who.int/topics/mortality/es/ Accessed March 25, 2020.

15. Soto-Estrada G, Moreno-Altamirano L, Pahua Díaz D, SotoEstrada G, Moreno-Altamirano L, Pahua Díaz D. Epidemiological overview of Mexico's lea ding causes of morbidity and mortality. Rev la Fac Med. 2016;59(6):8-22. http://www.scielo.org.mx/scielo.php?script=sci_arttext\&pid=S002617422016000600008 .

16. Rappaport SM. Genetic factors are not the major causes of chronic diseases. PLoS One. 2016;11(4):1-9. doi:10.1371/journal.pone. 0154387

17. Silverman EK. Applying functional genomics to chronic obstructive pulmonary disease. Ann Am Thorac Soc. 2018;15(4):S239S242. doi:10.1513/AnnalsATS.201808-530MG

18. Rathmann W, Scheidt-Nave C, Roden M, Herder C. Type 2 Diabetes: Prevalence and Relevance of Genetic and Acquired Factors for Its Prediction. Dtsch Arztebl Int. 2013;110(19):331-337. doi:10.3238/arztebl.2013.0331

19. Moore, Keith; Persaud, T; Torchia M. Embriología Médica. España: Elsevier; 2013

20. Greely HT. Human Germ line Genome Editing: An Assessment Cris J. 2019;2(5):253-265. doi:10.1089/crispr.2019.0038

21 03399448

https://clinicaltrials.gov/ct2/results?pg=1\&load=cart\&id=NCT

https://clinicaltrials.gov/ct2/results?pg=1\&load=cart\&id=NCT0339944 8. Accessed March 25, 2020.
22. Moses C, Kaur P. Applications of CRISPR systems in respiratory health: Entering a new 'red pen' era in genome editing. Respirology. 2019;24(7):628-637. doi:10.1111/resp.13527

23. German DM, Mitalipov S, Mishra A, Kaul S. Therapeutic Genome Editing in CardiovascularDiseases. JACC Basic to Transl Sci. 2019;4(1):122-131. doi:10.1016/j.jacbts.2018.11.004

24. Balboa D, Prasad RB, Groop L, Otonkoski T. Genome editing of human pancreatic beta cell models: problems, possibilities and outlook. Diabetologia. 2019;62(8):1329-1336. doi:10.1007/s00125019-4908-z

25. Lee H, Kim JS. Unexpected CRISPR on-target effects. Nat Biotechnol.2018;36(8):9-10. doi:10.1038/nbt.4207

26. Fu Y, Foden JA, Khayter C, et al. High-frequency off-target mutagenesis induced by CRISPR-Cas nucleases in human cells. Nat Biotechnol.2013;31(9):822-826. doi:10.1038/nbt.2623

27. Riveros Maidana R, Méndez Ferreira A, Benítez Candia N, Benítez Pereira E. Sistema CRISPR / Cas: Edición genómica de precisión. Memorias del Inst Investig en Ciencias la Salud. 2020;18(1):97-107.

28. Pérez-López J. Terapia génica en el tratamiento de los errores congénitos del metabolismo. Med Clin (Barc). 2014;142(12):549-553. doi:10.1016/j.medcli.2013.05.030

29. Bergel SD. Los Derechos Humanos: Entre La Bioética Y La Genética. Acta Bioeth. 2002;8(2):315-331. doi:10.4067/s1726$569 \times 2002000200011$

30. Fernandez Palacios P. EDICIÓN DE GENES MEDIANTE CRISPR-Cas9 : MÁS QUE CORTAR Y PEGAR. 2019.

31. Ivanov V. Good Gene/Bad Gene. (Boulyjenkov, V; Berg, K; Christen Y, ed.). Heidelberg; 2000. doi:https://doi.org/10.1007/978-3642-56947-0_14

32. Haldane JBS. The Part Played by Recurrent Mutation in Evolution. Am Nat. 1933;67:5-19. doi:10.2307/4609393

33. Verlarde Nañez M del P. Evolucion del papelde la Genetica en Medicina: del estudio de la herencia a la genomica personalizada. 2019.

34. McConnell SC. An Exclusive Interview with CRISPR. AMA J Ethics. 2019;21(1):1079-1088

35. Brokowski C, Adli M. CRISPR Ethics: Moral Considerations for Applications of a Powerful Tool. J Mol Biol. 2019;431(1):88-101. doi:10.1016/j.jmb.2018.05.044

36. Camara de Diputados S. Reglamento de La Ley General de Salud En Materia de Investigaciones Para La Salud.; 2014. http://www.cofepris.gob.mx/MJ/Paginas/Reglamentos.aspx.

37. López-Pacheco MC, Pimentel-Hernández C, Rivas-Mirelles E, Arredondo-Garcia JL. Normatividad que rige la investiga ción clinica en seres humanos y requisitos que debe cumplir un centro de investigación para participar en un estudio clinico en México. Acta Pediatr Mex. 2016;37(3):175-182. doi:10.18233/apm37no3pp175-182

38. Declaración de Helsinki de la AMM - Principios éticos para las investigaciones médicas en seres humanos - WMA - The World Medical Association. https://www.wma.net/es/policiespost/declaracion-de-helsinki-de-la-a mm-principios-eticos-para-lasinvestigaciones-medicas-en-seres-humanos/. Accessed March 26, 2020. 
39. Lecuona I De, Casado M, Marfany G, Baroni ML, Escarrabill M. Gene editing in humans: Towards a global and inclusive debate for responsible research. Yale J Biol Med. 2017;90(4):673-681.

40. Roland D. At \$ 2 Million, New Novartis Drug Is Priciest Ever. The Wall Street Journal.2019:1-5. 